\title{
WHAT SHOULD A \\ PSYCHIATRIST KNOW ABOUT \\ HORMONAL CONTRACEPTION?
}

\author{
Ana Margarida Mota1, Mariana Lázaro', Teresa Filipe1, Ana Cristina Farias1 \\ ${ }^{1}$ Centro Hospitalar Psiquiátrico de Lisboa, Portugal
}

\section{Backg rOUnd: Hormonal contraceptives $(\mathrm{HC})$ are widely used medications and adverse mood changes are a common complaint.}

Most of the research on psychological side effects of hormonal contraception is relatively scant and inconclusive.

\section{Impact on mood:}

O(Svendal 2012): Progestin-only contraceptive users had a three fold increased risk of a current mood disorder, compared to non-progestin users. In contrast, those currently using combined oral contraceptives (COC) were at decreased risk of a current mood disorder compared to non users.

(Skovlund 2016): Use of hormonal contraception, especially among adolescents, was associated with subsequent use of antidepressants and a first diagnosis of depression, suggesting depression as a potential adverse effect of hormonal contraceptive use.

O(Skovlund 2017): Use of hormonal contraception was positively associated with subsequent suicide attempt and suicide. Adolescent women experienced the highest relative risk.

(Pagano 2016): Limited evidence from six studies concluded that the use of the levonorgestrel-releasing intrauterine system (LNG-IUS) and depot medroxyprogesterone acetate (DMPA) was not associated with worse clinical outcomes, while COC tended to have a positive effect on mood.
(Merki-Feld 2017): Studies demonstrate that very few women complain of severe mood alterations as a consequence of using an LNG-IUS. In women who have already experienced major depression, the LNG-IUS and other progestin-only contraceptive methods are not the 1st choice and close clinical follow-up is necessary.

(Oinonen, 2001): While the only consistent OC-related mood effects experienced by most women are beneficial, a subgroup do experience negative mood change.

\section{No effect on mood:}

O(Schaffir 2016): Inconsistent research methods and lack of uniform assessments make it difficult to make strong conclusions about which users are at risk for adverse mood effects. Clinicians should recognise that such effects are infrequent and $\mathrm{CHC}$ may be prescribed with confidence.

(McKetta 2018): This study found no increased risk of depressive disorders among oral contraceptive pills users.

(Pagano 2016): evidence from 1 RCT and 5 prospective cohort studies suggests that COC use among women with depressive or bipolar disorders was not associated with worse clinical course compared with no hormonal method use.

\section{Despite these mixed results...}

...certain findings offer some

clinical insights, such as:

\section{Women with underlying mood disorders may be predisposed to mood effects;}

\section{Progestin-only contraceptives may have a negative influence on mood, whereas estrogens may have positive effects on depressive symptoms;}

\section{Continuous dosing of $\mathrm{HC}$ has the fewest mood effects.}

\section{Conclusion:}

Based on the current knowledge, decisions regarding starting or discontinuing HC should be made on a individual basis.

Contraceptive counselling:

OCarry out a biopsychosocial assessment of the woman, including her mental condition and environmental distress, as part of her psychosocial and cultural profile.

OProvide a choice of contraceptive methods, including the use of different progestogens with differential actions on different parts of the body such as the brain.

ODuring use, ask proactively about side effects, including questions about sexual function and affective symptoms, and explore factors contributing to any complaints that may be due to the contraceptive used or to other causes. 